\section{Simplified Selection of Transgenic Arabidopsis thaliana Seed in Liquid Culture}

BioTechniques 22:62-63 (January 1997)

We have developed a technique that simplifies the process of screening large quantities of Arabidopsis thaliana seeds to identify antibiotic-resistant individuals. We use this technique in our laboratory for screening seed populations after transforming by vacuum infiltration (1). Conventional screening methods are material- and labor-intensive, particularly if transformation frequencies are low, due to the necessity of plating large amounts of seeds at densities low enough to observe antibiotic-resistant plants. We minimize these difficulties by germinating seeds in liquid media.

Germinating seeds in liquid culture makes it fairly simple for us to sterilize and grow a large amount of seeds. However, in standard liquid culture media, seeds aggregate rapidly. As they germinate, a jumble of plant tissue results, making it impossible to remove plants of interest for further growth on soil. The key to the success of our technique is the supplementation of the growth media with $0.1 \%$ agar before autoclaving. Upon cooling, a suspension of fine agar particles forms, and this suspension interferes with the seeds' natural tendency to aggregate.

Eighteen thousand $A$. thaliana ecotype Wassilewskija (WS) seeds ( $0.36 \mathrm{~g})$ were surface-sterilized (30-min imbibition with water, followed by $5 \mathrm{~min}$ in $95 \%$ ethanol, 5 min in $10 \%$ bleach and 5 rinses with sterile water) as described in (2) and added to each of three 2-L flasks with $4 \mathrm{~mL}$ of germination media [1× MS salts (3) plus $1 \%$ (wt/vol) sucrose, $100 \mathrm{mg} / \mathrm{L}$ myoinositol, $1.0 \mathrm{mg} / \mathrm{L}$ thiamine, $0.5 \mathrm{mg} / \mathrm{L}$ pyridoxine, 0.5 $\mathrm{mg} / \mathrm{L}$ nicotinic acid, $0.5 \mathrm{~g} / \mathrm{L}$ 2-(morpholino)ethanesulfonic acid (MES), $\mathrm{pH}$ 5.65-5.8 with potassium hydroxide]. Ten seeds carrying an insert conferring kanamycin resistance (pBI121; CLONTECH Laboratories, Palo Alto, CA, USA) were added to one of the flasks. After a two-day cold treatment at $4^{\circ} \mathrm{C}$, $250 \mathrm{~mL}$ of germination media were added to each flask. One of the flasks containing only wild-type WS seeds received standard germination media; the other two flasks received germination media supplemented with $0.1 \%$ agar (Bacto-Agar; Difco Laboratories, Detroit, MI, USA). Kanamycin (75 $\mu \mathrm{g} / \mathrm{mL}$ ) was added to the flask containing transgenic seeds. As an extra precaution, Timentin ${ }^{\circledR}(100 \mu \mathrm{g} / \mathrm{mL}, 30: 1$ ticarcillin:clavulanic acid; SmithKline Beecham, Philadelphia, PA, USA) was added to reduce bacterial contamination, but it may not be strictly necessary. Flasks were placed on a rotating platform and shaken at $70 \mathrm{rpm}$ under fluorescent lights $(40 \quad \mu \mathrm{E}$ $\mathrm{m}^{-2} \mathrm{~s}^{-1}$ ) and photographed after seven days of growth.

Figure 1A shows the effect of agar

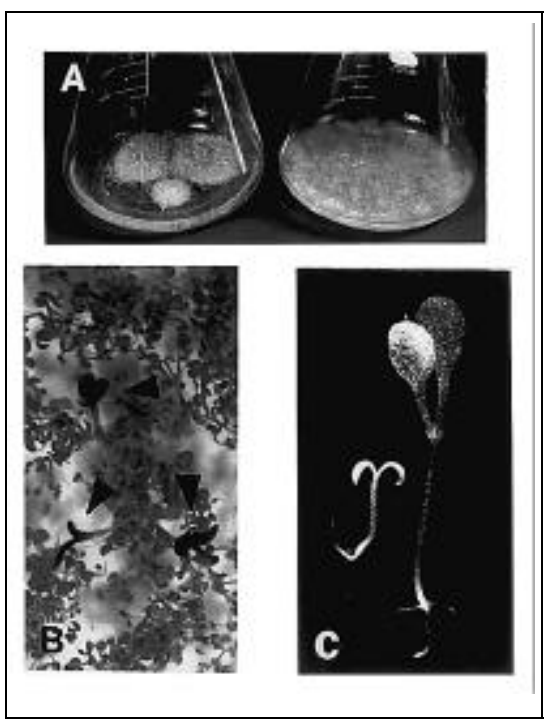

Figure 1. Seedling growth under selected conditions. (A) Seedlings grown without agar (left) and with agar (right). (B) High magnification view of liquid culture with antibiotics showing darker kan ${ }^{\mathrm{r}}$ seedlings (arrowheads). (C) Comparison of $\mathrm{kan}^{\mathrm{s}}$ (left) vs. $\mathrm{kan}^{\mathrm{r}}$ (right) seedlings. 
on seedling growth in the absence of antibiotic. The flask on the left shows WS seeds germinated in media without added agar, growing into large tangled clumps. The flask on the right shows the effect of the addition of $0.1 \%$ agar-the seedlings are now growing separately.

Figure 1B shows the flask containing a mixture of wild-type WS plants and kanamycin-resistant $\left(\mathrm{kan}^{\mathrm{r}}\right)$ plants. The wild-type, kanamycin-sensitive $\left(\mathrm{kan}^{\mathrm{s}}\right)$ plants have bleached to yellow, while the $\operatorname{kan}^{\mathrm{r}}$ plants are dark green and are easily retrieved. Nine of the ten transgenic seedlings were recovered in this example. The close-up photograph in Figure $1 \mathrm{C}$ demonstrates the marked size difference between the $\mathrm{kan}^{\mathrm{s}}$ and resistant seeds.

We have made several observations on variations of this technique that may be helpful. Kanamycin-sensitive seedlings grow to different final sizes under different light levels. Under lower light, they elongate more, so the size difference between sensitive and resistant seeds is not as pronounced. However, the resistant seedlings are still easily identified by their dark green color. Resistant seedlings also exhibit apical shoot growth and produce secondary roots. Under higher light, the hypocotyls curve more, which leads to the formation of many small clumps as seedlings hook together. These clumps disperse with continued shaking, and once again resistant seedlings are easily isolated. We generally use a $2-\mathrm{L}$ flask and adjust the rotation speed to produce a slow smooth traveling wave. The rotation speed should be adjusted for different sized flasks to maintain an even suspension of seeds. Antibioticresistant seedlings are recovered by emptying the contents of the flask into a glass baking dish and removing resistant plants with forceps. When antibiotic-resistant seedlings are transferred to plates or soil, the first 2 to 4 leaves are usually deformed, but subsequent leaves return to wild-type morphology.

In summary, we have developed a method that allows us to germinate $A$. thaliana seeds in liquid culture without forming a large inseparable clump of seedlings. The addition of $0.1 \%$ agar prevents this clumping. When combined with kanamycin selection, kan ${ }^{\mathrm{r}}$ seedlings can be easily identified and retrieved. The simplicity of this technique and the time and materials that it saves make it very useful for screening large quantities of seeds for transformants.

\section{REFERENCES}

1.Bechtold, N., J. Ellis and G. Pelletier. 1993. In planta Agrobacterium mediated gene transfer by infiltration of adult Arabidopsis thaliana plants. Comptes Rendus de l'Academie des Sciences Serie III Sciences de la Vie 316:1194-1199.

2.Lehle Seeds. 1993. Lehle Seeds Arabidopsis Catalog. Round Rock, TX.

3.Murashige, T. and F. Skoog. 1962. A revised medium for rapid growth and bioassays with tobacco tissue cultures. Physiologia Plantarum 15:473-497.

This research was supported by grants from the University of Wisconsin-Madison Graduate School and NSF Grant No. DCB9105527 to D.E.F and by grants to the University of Wisconsin-Madison from the DOE/NSF/USDA Collaborative Program on Research in Plant Biology (Grant No. BIR 92-20331). Address correspondence to Donna E. Fernandez, Department of Botany, University of Wisconsin, 430 Lincoln Drive, Madison, WI 53706-1381, USA. Internet:dfernand@facstaff.wisc.edu

Received 29 February 1996; accepted 24 May 1996.

Karl W. Nichols, Gregory R. Heck and Donna E. Fernandez University of WisconsinMadison Madison, WI, USA 